Muñoz Cantero, Jesús Miguel; Rebollo Quintela, Nuria (2016). Evaluación del impacto de políticas orientadas a la inserción sociolaboral mediante matching. Revista de Investigación Educativa, 34(1), 87-102.

DOI: http://dx.doi.org/10.6018/rie.34.1.206871

\title{
Evaluación del impacto de políticas orientadas a la inserción sociolaboral mediante matching*
}

\author{
Impact assessment of policies for the sociolabor \\ insertion trough matching
}

\author{
Jesús Miguel Muñoz Cantero y Nuria Rebollo Quintela \\ Universidad de A Coruña
}

\begin{abstract}
Resumen
La Conferencia sobre Políticas activas de empleo para la Europa de 2020 delimitó dentro de las 10 "maneras de avanzar" dos propuestas que destacan la importancia de realizar una evaluación sistemática a fin de determinar la eficacia de las políticas activas - (1) Los Estados miembro deberían hacer más para evaluar la eficacia de sus politicas activas - , - (2) Con el fin de tener un mayor impacto, la nueva Estrategia Europea de Empleo necesita una fuerte dimensión de "evaluación" -. Uno de los objetivos principales que se pretende alcanzar con la puesta en marcha de las políticas activas de fomento del empleo, es la inserción laboral de sus participantes. En este estudio, se pretende determinar el impacto del programa de inserción sociolaboral "Puesto a Puesto VII" a través de un análisis matching. Los resultados obtenidos permiten acreditar el impacto del programa en las variables resultado.

Palabras clave: estrategia europea de empleo, política de empleo, evaluación, matching.

Correspondencia: Dr. D. Jesús Miguel Muñoz Cantero. Universidad de A Coruña. Campus de Elviña s/n, 15071, A Coruña. jesus.miguel.munoz@udc.es

Dra. Dña. Nuria Rebollo Quintela. Universidad de A Coruña. Campus de Elviña s/n, 15071, A Coruña. nuria.rebollo@udc.es

* Investigación fruto de la tesis doctoral financiada a través de la ayuda de apoyo a la etapa predoctoral del Plan Galego de Investigación, Innovación e Crecemento 2011-2015 Xunta de Galicia (Plan I2C)
\end{abstract}




\begin{abstract}
The Conference Active Labour Market Policies for Europe 2020 finding "ways to move forward" delineated two proposals that emphasize the importance of a systematic evaluation to determine the effectiveness of active labor market policies - (1) Member States should make more efforts to evaluate their ALMPS -, - (2) In order to have more impact, there is a need for a strongly developed evaluation dimension within the new European Employment strategy -. One of the main objectives to be achieved with the implementation of active policies to promote employment is the employment of its participants. This study aims to determine the impact of labor insertion program "Puesto a Puesto VII" through a matching analysis. The results obtained demonstrate the impact of the program on outcome variables.

Keywords: European Employment Strategy, employment policy, evaluation, matching.

\section{Introducción}

La evaluación de las iniciativas de los Estados Miembros de la Unión Europea en materia de políticas de empleo es de gran importancia para determinar en qué contexto y con qué mecanismos deben ser aplicadas (Alonso-Borrego, Arellano, Dolado y Jimeno, 2004). Una vez articuladas, a través de ellas y de sus redes de actores, se podrá establecer cuáles se van a poder desarrollar a nivel europeo y a nivel nacional para tener una visión general de las reformas del mercado de trabajo necesarias que ayudarán a fijar sus puntos críticos de acuerdo con los diferentes modelos de bienestar social existente en la Europa Comunitaria (González-Blanch, 2010).

Así mismo, la puesta en marcha de programas de formación y asesoramiento, ha supuesto la asunción por parte del Consejo Europeo de mecanismos de evaluación, estableciendo una serie de indicadores comunes para todos los países a fin de poder realizar una evaluación de las medidas tanto a nivel nacional como europeo (Monclús y Sabán, 2000). Este hecho subraya la importancia de un seguimiento más efectivo de las estrategias de empleo, a fin de alcanzar mayor eficacia en la intervención y el intercambio de experiencias en el marco comunitario.
\end{abstract}

\title{
Revisión teórica del tema investigado
}

\section{La evaluación de las políticas públicas en la Unión Europea}

La finalidad de la evaluación de las políticas públicas en la Unión Europea ha mudado en las últimas décadas para responder al contexto socioeconómico y político donde se desarrollan. Zapico-Goñi (2010) alude a que nace bajo una concepción centrada en la gestión por resultados para ir derivando en lo que Berman (1980) denomina aproximación adaptativa participativa.

A modo de síntesis Moreno y Ziglio (como se citó en Herrador Buendía, 2008) exponen nueve razones y finalidades del uso evaluativo en las políticas públicas activas del mercado de trabajo (tabla 1). 
Tabla 1

Razones y finalidades de la evaluación

\section{¿Por qué la evaluación?}

Para demostrar a los demás que la política o el programa es/ ha sido acertado

Para determinar si un programa va por el buen camino

Para determinar la efectividad del programa a fin de satisfacer las necesidades por las cuales se creó

Para justificar gastos presupuestarios o proyectados

Para determinar los costes de un programa relativos a los recursos humanos o financieros

Para apoyar la expansión de un programa

Para comparar diversos tipos de programas en términos de eficacia/eficiencia

Para satisfacer las demandas de los funcionarios responsables de los servicios sociales, los cuales exigen pruebas de los efectos de los programas

Para retrasar la decisión de poner en marcha una nueva política o programa mediante la petición de evaluaciones y pruebas que justifiquen la efectividad de dicha política o programa

Nota: Adaptado de Política social de Mercado de trabajo en España. El origen del proceso de europeización de la política de fomento del empleo,(p.58) por F. Herrador Buendía, 2008,Valencia:Tirant lo Blanch

Nos encontramos actualmente, con un discurso europeísta que gira en torno a la necesidad de desarrollar procesos de evaluación para mejorar la calidad y la eficacia de las políticas públicas. Esta mejora de la calidad se pretende alcanzar en base a las siguientes propuestas enunciadas por la Comisión Europea en 2001(Zapico-Goñi, 2010, p. 108):

- Reforzar la cooperación institucional (Parlamento Europeo- Consejo de Ministros- Comisión-Europea) para la evaluación y desarrollar una colaboración más estrecha con los Estados Miembros y la sociedad.

- Aumentar la capacidad de evaluación de la Comisión y los Estados Miembros.

- Desarrollar instrumentos y procedimientos participativos para evaluar la acción legislativa y presupuestaria de la Unión Europea.

- Mejorar los sistemas de seguimiento y disponibilidad de datos empíricos en los Estados Miembros.

- Integrar la evaluación en las funciones de planificación y presupuestos; aumentar la transparencia y difusión proactiva de los resultados de la evaluación.

- Construir una cultura de resultados que conlleve una toma de decisiones basada en el análisis más que en concesiones ad hoc.

Somos conscientes que los resultados en una política activa de empleo determinada pueden ser considerados como un hecho aislado, que no suscite un cambio en el modelo 
instaurado en la Unión Europea, pero si estos resultados se dan en varios programas, sobre todo detectando sus debilidades, influenciaran las decisiones tomadas por los responsables políticos y administrativos (Ballart Hernández, 2005).

La evaluación de las políticas activas de empleo, y concretamente de los programas de formación, tiene un largo recorrido en Estados Unidos y Europa, pero en España no ha sido hasta la década de los 90 que se ha experimentado un aumento de la cultura evaluadora, ejemplo de ello son las investigaciones de Alujas Ruíz y López Tamayo (2006), Ayala y Rodríguez (2006, 2007, 2011), Buendía Eisman, Expósito López y Sánchez Martínez (2012), de Miguel, San Fabián, Belver y Argüelles (2011), García Pérez y Rebollo Sanz (2009a, 2009b), Olmos Rueda (2014), Rebollo Quintela y Muñoz Cantero (2013) y Suárez Cano, Mayor Fernández y Cueto Iglesias (2011).

La evaluación de las políticas activas de empleo viene justificada por la necesidad de determinar la efectividad y la eficacia. La efectividad en el logro de los objetivos propuestos y la eficacia en términos de relación entre el logro de objetivos y recursos invertidos para alcanzarlos. Además sólo a través de un análisis detallado de los programas se determinará sobre qué poblaciones tendrán una mayor incidencia y cuáles van a ser los mejores procesos a llevar a cabo para su correcta puesta en marcha y ejecución. De hecho en la Conferencia sobre Políticas activas de empleo para la Europa de 2020 se delimitó dentro de las 10 "maneras de avanzar" 2 propuestas que destacan la importancia de realizar una evaluación sistemática a fin de determinar la eficacia de las políticas activas - (1) Los Estados miembro deberían hacer más para evaluar la eficacia de sus políticas activas - , - (2) Con el fin de tener un mayor impacto, la nueva Estrategia Europea de Empleo necesita una fuerte dimensión de "evaluación" -. Jornet et al. (2001), aluden a que la evaluación de la formación para el empleo es un instrumento fundamental para la mejora de la calidad de los programas.

\section{Modelos de evaluación}

Existen diferentes enfoques y modelos que abordan la evaluación de las políticas activas de empleo a nivel nacional e internacional, y más en concreto, las políticas de formación y asesoramiento, entre ellos los que han cobrado mayor relevancia es la evaluación de la eficacia desde el punto de vista de los efectos que se pueden desencadenar con la puesta en marcha de estas medidas, pudiendo ser estos, como afirma García Serrano (2007) a nivel macroeconómico o a nivel microeconómico:

- Los efectos a nivel macroeconómico se refieren a aquellos que afectan a la economía en su conjunto, si la realización de estas acciones influyen tanto de manera positiva como negativa sobre indicadores agregados del mercado de trabajo como la tasa de empleo, de participación y de paro, ... relacionando estas variables con indicadores del grado de intensidad de los programas de empleo (número de trabajadores cubiertos, gasto por trabajador afectado por los programas,...).

- Los efectos a nivel microeconómico afectarán directamente a los agentes económicos individuales. Se centran en la medición del efecto de los programas sobre la probabilidad de salida del paro (obtención de un trabajo) o sobre la calidad del empleo y salarios futuros de los trabajadores beneficiarios de los programas. 


\section{Evaluaciones de programas de formación y asesoramiento}

Los resultados hallados en los estudios sobre evaluaciones de las políticas activas a nivel nacional y internacional centrado en programas de formación y asesoramiento a la búsqueda de empleo, aunque dispares son coincidentes en las conclusiones generales extraídas. Se evidencia que la participación en los programas de formación tienen un efecto positivo y significativo sobre la salida del desempleo (Alonso Ramos, 2005; 2008; Anxo, Carcillo, y Erhel, 2001; Boeri y Burda, 1996; de Koning y Arents, 2001; de Miguel, San Fabián, Belver y Argüelles, 2012; Meager y Evans, 2002; Meyer, 2000; Richardson y Berg, 2001), siendo este mayor a corto plazo, pero no influyen en la salida del paro hacia la ocupación (García Serrano, Malo, Herranz, Davia, y Toharia, 2001; Mato Díaz, 2002).

Rocha Sánchez (2010) en una recopilación de evaluaciones de medidas de fomento del empleo, puestas en marcha tras la crisis de 2008, alude a un impacto positivo de estas acciones para la reducción del paro, así como para el aumento de la ocupación, si bien reconoce que su alcance es limitado y que dependerá en gran medida de su puesta en marcha junto con otras políticas que contribuyan a la dinamización económica y, por ende, productiva.

Los estudios referenciados, usan diferentes métodos para calcular el impacto de las medidas de formación y asesoramiento, métodos experimentales, cuasi experimentales, observacionales.

En Europa son más frecuentes las evaluaciones de corte no experimental, frente a las experimentales usadas en EEUU. En las no experimentales, al no haber un grupo control propiamente dicho, se conforma uno con los no participantes, con similares características a los participantes, partiendo de unos supuestos que nos permitan eliminar los sesgos de selección (diferencias entre el grupo tratamiento y el grupo control).

\section{El método matching}

Aunque existen diferentes modelos para estimar el efecto causal de una política de empleo, el método matching ha cobrado una gran relevancia en los estudios observacionales en las últimas décadas en diferentes campos desde que fueron introducidos por Rosenbaun y Rubin en 1983 (Affuso, 2010).

Desde este punto de vista respondemos a la pregunta ¿cuál es el efecto del tratamiento? O lo que es lo mismo, ¿cuál es el efecto causal de una determinada política, sobre la variable que queremos medir? Lo que se pretende es que los resultados obtenidos describan la situación laboral de los beneficiarios de un determinado programa, grupo de tratamiento, transcurrido un período de tiempo relativamente corto (de entre 6 a 18 meses). Sin embargo, nos encontramos con el problema del desconocimiento de hasta qué punto el cambio en la situación laboral del participante viene determinado por la participación en el programa, si no probamos el efecto causal (Cueto Iglesias y Mato Díaz, 2004). Efecto causal entendido en términos de variación en la variable objeto de estudio que se debe exclusivamente al tratamiento y no a otros factores. 
En el método matching las diferencias se producen en características observables (formación, años trabajados, ingresos,...) por lo que si el emparejamiento se realiza con otra persona con similares características del grupo control, las diferencias en las variables estudiadas se deberán exclusivamente al tratamiento (Arellano, 2006).

Por lo tanto, para un participante el impacto se mide como la diferencia entre el valor de su variable resultado en su situación actual $\left(\mathrm{Y}_{1}\right)$, y el valor de su resultado contrafactual en la situación hipotética de que no hubiera participado en el programa $\left(Y_{0}\right)$. Por lo tanto se determinaría qué habría ocurrido de no haber participado. El efecto del tratamiento en el individuo se calcularía como la diferencia entre los dos resultados, siendo 1 la participación en un programa (tratamiento) y 0 la no participación, expresados como $\Delta=\left(\mathrm{Y}_{1}-\mathrm{Y}_{0}\right)$. Siendo $\Delta$ el impacto del programa.

Como no es posible conocer $\mathrm{Y}_{1}$ e $\mathrm{Y}_{0}$ para el mismo sujeto, surge la necesidad de la estimación del contrafactual, un grupo de comparación adecuado, a partir del cual estimar el resultado sin participación (Mato Díaz y Cueto Iglesias, 2008).

El efecto del tratamiento sobre los tratados, nos permite determinar el impacto de un programa sobre el grupo específico de sujetos al que se le ha aplicado (Palma Martos, Borra Marcos, González Camacho y Aguado Quintero, 2009), donde D es una variable dummy que indica la participación (1) o no participación (0) en el programa:

$$
\Delta_{\text {ATT }}=E\left(Y_{1}-Y_{0} \mid X, D=1\right)=E(\Delta \mid X, D=1)
$$

Para lo que necesitaríamos conocer el efecto de los beneficiarios de no haber participado, $\mathrm{E}\left(\mathrm{Y}_{0} \mid \mathrm{X}, \mathrm{D}=1\right)$, que se soluciona con la construcción del contrafactual.

Existen diferentes métodos y modelos que proporcionan opciones para construir el contrafactual, que pretenden la eliminación o disminución en su mayoría del sesgo de selección, mediante la elección del grupo control (Durán, 2004):

- Encontrando individuos idénticos a los participantes con respecto a todas las características relevantes excepto la participación en el programa de formación.

- O se controla todas las diferencias relevantes que existen entre los participantes y los no participantes.

La comparación entre el grupo tratamiento y el grupo control permite determinar el efecto medio del tratamiento más un sesgo de selección (donde el error de selección aparece entre corchetes), ya que si no los efectos del tratamiento se podrían deber a las diferencias en las características previas entre unos y otros.

$$
\begin{gathered}
\mathrm{E}[\mathrm{Y} \mid \mathrm{D}=1]-\mathrm{E}[\mathrm{Y} \mid \mathrm{D}=0]=\mathrm{E}\left[\mathrm{Y}_{1} \mid \mathrm{D}=1\right]-\mathrm{E}\left[\mathrm{Y}_{0} \mid \mathrm{D}=1\right]= \\
\mathrm{E}\left[\mathrm{Y}_{1}-\mathrm{Y}_{0} \mid \mathrm{D}=1\right]+\left\{\mathrm{E}\left[\mathrm{Y}_{0} \mid \mathrm{D}=1\right]-\mathrm{E}\left[\mathrm{Y}_{0} \mid \mathrm{D}=0\right]\right\}
\end{gathered}
$$

A través del matching o emparejamiento se restablecerán las condiciones de un experimento mediante la construcción de un grupo de comparación similar al grupo tratamiento, con ello se elimina el sesgo de selección al condicionarlo a las variables observables (Palma Martos et al., 2009). 
Las maneras de reducir el sesgo de selección serán (Mato Díaz y Cueto Iglesias, 2008):

- La comparación de individuos comparables entre aquellos que se encuentren en la región común (common support).

- La ponderación de cada individuo para que ambos grupos, control y tratamiento, tengan la misma distribución de variables observables.

- Obtención de datos lo más completos posibles, a fin de reducir el sesgo basado en variables inobservables.

El problema de eliminar el sesgo de selección condicionándolo a las características observables surge cuando tenemos muchas variables (sexos, edad, nivel de estudios, estado civil, experiencia laboral, tiempo en desempleo,...), y para solucionar esta dimensionalidad Rosenbaum y Rubin (como se citó en Cueto Iglesias y Mato Díaz, 2004) propusieron condicionar en el propensity score o puntuación de asignación

El matching asume el supuesto de independencia condicionada donde la adopción del tratamiento es independiente de los valores potenciales de la variable objeto de estudio Y, dadas las características observables X (Arellano, 2006).

$$
\left(Y_{0}, Y_{1}\right) D \mid X
$$

Siendo la probabilidad de participar en el programa (propensity score) (Durán, 2004):

$$
\mathrm{P}(\mathrm{X})=\operatorname{pr}(\mathrm{D}=1 \mid \mathrm{X})
$$

Teniendo en cuenta los supuestos enunciados "el resultado de la participación en un programa es el mismo para participantes y no participantes, una vez controlado por las variables $X$ o por la probabilidad de asignación $\mathrm{P}(\mathrm{x})^{\prime \prime}$ en base a lo demostrado por Rosenbaum y Rubin (como se citó en Mato Díaz y Cueto Iglesias, 2008, p. 65), si:

$$
\begin{gathered}
\left(\mathrm{Y}_{0^{\prime}}, \mathrm{Y}_{1}\right) \mathrm{D} \mid \mathrm{X} \text { y } 0<\mathrm{P}(\mathrm{x})<1 \text {, donde } \mathrm{P}(\mathrm{x})=\operatorname{pr}(\mathrm{D}=1 \mid \mathrm{X}) \text { entonces } \\
\left(\mathrm{Y}_{0^{\prime}}, \mathrm{Y}_{1}\right) \mathrm{D} \mid \mathrm{P}(\mathrm{x})
\end{gathered}
$$

Una vez calculado el propensity score ya podemos estimar el efecto medio del tratamiento sobre los tratados (ATT):

$$
\begin{aligned}
& \mathrm{ATT}=\sum\left\{\mathrm{y}_{\mathrm{i}}-\hat{\mathrm{y}}_{\mathrm{i}}\right\} \mathbf{w}_{\mathrm{i}} \\
& \operatorname{iv}\left\{\mathrm{D}_{\mathrm{i}} \cap \mathrm{S}_{10}\right\}
\end{aligned}
$$

$\mathrm{y}_{\mathrm{i}}$ es el resultado para un individuo i del grupo de tratamiento, mientras que $\hat{y}_{\mathrm{i}}$ es el resultado del individuo del grupo de comparación con el que se realiza el emparejamiento calculado como:

$$
\underset{j v C^{0}\left(p_{i}\right)}{\hat{y}_{i}=\sum w_{i j} y_{i}}
$$


$\mathrm{C}^{0}\left(\mathrm{p}_{\mathrm{i}}\right)$ grupo de observaciones vecinas del individuo i del grupo de tratamiento en el grupo control, $w_{\text {ij }}$ ponderación de la observación j del grupo control para formar una unidad comparable a la observación i del grupo tratamiento (Blundell, Dearden, Sianesi, 2004).

$\mathrm{S}_{10}$ región común

$\mathbf{w}_{\mathrm{i}}$ ponderación igual a $\left[1 /\right.$ número de tratados en $\left.S_{10}\right]$

De lo que se deriva que "el efecto medio del tratamiento para los tratados se calcula como la diferencia entre el resultado para los individuos del grupo tratamiento y del grupo control, teniendo en cuenta el número de individuos en el rango común" (Cueto Iglesias y Mato Diaz, 2005, p.18)

Existen diferentes métodos matching, algunos especialmente útiles cuando se usan variables continuas debido a la dificultad de encontrar dos observaciones con el mismo valor, ya que estos métodos nos permitirán realizar emparejamientos con una media ponderada de observaciones del grupo de comparación con reemplazamiento o no. Si se permite el reemplazamiento, una unidad del grupo control puede ser pareja de varias del grupo tratamiento, lo que reducirá el sesgo ya que aumenta el número de pares posibles (Abadie e Imbens, 2006).

Autores como Dehejia y Wahba (2002) aluden a los beneficios del propensity score matching para reproducir los resultados del análisis experimental, sin embargo Smith y Todd (2005) muestran sus limitaciones ya que los resultados son muy sensibles tanto a la selección de la muestra como a la de las variables usadas para el cálculo del propensity score.

\section{Objetivos}

El objetivo de este estudio es analizar la eficacia del programa "Puesto a Puesto VII", programa tutorizado de inserción laboral diseñado e implantado en el Centro Municipal de Empleo del Ayuntamiento de A Coruña para el colectivo femenino. A través del análisis de la eficacia, pretendemos determinar si el programa supone una mejora, medida en las dos variables resultado (acceso al empleo y tiempo trabajado) en el grupo tratamiento respecto al grupo control.

\section{Metodología de investigación}

\section{Participantes}

La investigación se realizó con 144 participantes, 46 del grupo control y 98 del grupo tratamiento. A continuación se detalla el proceso seguido para conformación de ambos grupos:

En primer lugar, para poder llevar a cabo el programa evaluado, "Puesto a Puesto VII" el Servicio Público de Emprego de Galicia envió cartas a 200 mujeres que en ese momento se encontraban en desempleo, convocándolas a entrevistas grupales en el Centro Municipal de Empleo. Este servicio citó, en grupos de veinte personas, a las demandantes de empleo, para que les pudiéramos explicar las características del 
programa. Tras las reuniones grupales se las dio una cita individual a cada una de ellas, para una valoración de sus necesidades y su adecuación al programa. De las 200 mujeres, 18 no tenían interés en participar en el proceso de selección del programa por diferentes motivos personales y de salud. Este aspecto es de destacar ya que reduce el sesgo de selección en las características inobservables basado en la voluntariedad en participar en el programa (Raaum y Torp, 2002). La selección del grupo tratamiento frente al control fue por orden de cita en las entrevistas individuales. Conforme se le realizaba la entrevista, si estaban dispuestas a participar en el programa, firmaban la aceptación y entraban a formar parte del mismo. El programa estaba diseñado para 120 mujeres por lo que finalmente, el grupo control lo forman 62 sujetos.

\section{Instrumento}

La información sobre el impacto del programa se recopiló a través de dos entrevistas semiestructuradas (una a las beneficiarias del programa y otra al grupo control), diseñadas adhoc y para las que se tuvo en cuenta el instrumento elaborado por Cueto Iglesias y Mato Díaz (2005).

\section{Procedimiento}

Para la recogida de la información se realizaron entrevistas telefónicas durante los meses de Diciembre de 2013 a Marzo de 2014, un año después de la finalización del programa. Las entrevistadas fueron informadas de los objetivos de la investigación así como de la confidencialidad de sus datos.

\section{Resultados}

En primer lugar conviene definir de forma comparativa aquellas variables de la muestra, características observables en las que posteriormente vamos a condicionar el propensity score.

La variable edad no difiere entre el grupo tratamiento y el grupo control, ya que la media es de 42 años.

El nivel de formación, presenta diferencias en la categoría sin estudio ya que en el grupo control no existe representación de esta, frente al 6.12\% del tratamiento. Sin embargo, el porcentaje de universitarios es mayor en el grupo control. En las categorías formativos medias (Graduado Escolar/ EGB/ ESO, BUP/ COU/ Bachillerato y FPs) se observan resultados similares.

Comparativamente, cobran prestación un mayor número de sujetos en el grupo tratamiento que en el control con una diferencia porcentual de 3.3 puntos. Las beneficiarias del programa que cobran desempleo representan un $50 \%$, frente al grupo de comparación que lo hacen en un $21.73 \%$. Mismo porcentaje que representa la ayuda familiar en el grupo control, frente al 15.3\% del grupo tratamiento.

Por último, el tiempo en desempleo de la muestra de beneficiarias es inferior a la del grupo tratamiento, ya que la categoría predomínate es la de menos de 1 año $(46.94 \%)$, frente a los más de 2 años en desempleo de la muestra del grupo control (43.47\%). 
Tabla 2

Estadísticos descriptivos de los grupos tratamiento y control

\begin{tabular}{|c|c|c|c|c|c|c|}
\hline \multicolumn{7}{|c|}{ Estadísticos descriptivos de los grupos tratamiento y control } \\
\hline & \multirow{2}{*}{\multicolumn{2}{|c|}{$\begin{array}{c}\text { Grupo } \\
\text { tratamiento } \\
\mathrm{n} 98\end{array}$}} & \multicolumn{2}{|c|}{ Grupo control } & \multicolumn{2}{|c|}{ Muestra total } \\
\hline & & & \multicolumn{2}{|c|}{ n 46} & \multicolumn{2}{|c|}{ N 144} \\
\hline & M & SD & M & SD & M & SD \\
\hline Edad (años) & 42.29 & 9.64 & 42.60 & 10.90 & 42.39 & 10.03 \\
\hline \multicolumn{7}{|l|}{ Estudios (\%) } \\
\hline Sin Estudios & 6.12 & 0.24 & 0 & 0 & 4.16 & 0.20 \\
\hline Graduado Escolar/ EGB/ESO & 33.67 & 0.47 & 34.78 & 0.48 & 34.02 & 0.47 \\
\hline BUP/COU /Bachillerato & 16.32 & 0.37 & 15.21 & 0.36 & 15.97 & 0.36 \\
\hline FPs & 35.71 & 0.48 & 30.43 & 0.46 & 34.02 & 0.47 \\
\hline Universitarios & 8.16 & 0.08 & 19.56 & 0.40 & 11.80 & 0.32 \\
\hline No cobran prestación (\%) & 20.4 & 0.40 & 17.39 & 0.38 & 19.44 & 0.39 \\
\hline Cobran prestación (\%) & 79.6 & 0.40 & 73.91 & 0.44 & 77.77 & 0.41 \\
\hline Desempleo/ Paro & 50 & 0.50 & 21.73 & 0.41 & 40.97 & 0.49 \\
\hline Mayores de 45 & 1.02 & 0.10 & 8.7 & 0.28 & 3.47 & 0.18 \\
\hline Mayores de 52 & 7.14 & 0.25 & 6.52 & 0.25 & 6.94 & 0.25 \\
\hline Recualificación profesional & 3.06 & 0.17 & 4.34 & 0.20 & 3.47 & 0.18 \\
\hline Ayuda familiar & 15.3 & 0.36 & 21.73 & 0.41 & 17.36 & 0.38 \\
\hline Otras & 3.06 & 0.17 & 10.86 & 0.31 & 5.55 & 0.23 \\
\hline Experiencia laboral previa (\% sí) & 100 & 0 & 97.82 & 0.14 & 99.30 & 0.08 \\
\hline Meses de experiencia laboral & 137.86 & 91.86 & 134.91 & 94.34 & 136.93 & 92.32 \\
\hline \multicolumn{7}{|l|}{ Tiempo en desempleo (\%) } \\
\hline Menos de 1 año & 46.94 & 0.50 & 30.43 & 0.46 & 41.66 & 0.49 \\
\hline Entre 1 y 2 años & 33.67 & 0.47 & 23.91 & 0.43 & 30.55 & 0.46 \\
\hline Más de 2 años & 19.39 & 0.39 & 43.47 & 0.50 & 27.08 & 0.44 \\
\hline
\end{tabular}

Ambos grupos (tratamiento y control) los conforman sujetos con características diferentes, pero esas diferencias son controlables a través del método matching que nos permitirá determinar la eficacia del programa evaluado.

En un primer lugar se realiza el cálculo del propensity score a través de un modelo probit, que nos indica la probabilidad que tiene una persona de ser aceptada en el programa.

La edad, el nivel de formación y la experiencia laboral previa no actúan como variables significativas a la hora de acceder al programa, sin embargo en base al modelo, haber cobrado prestación aumenta las posibilidades de acceder al programa. 
No obstante, la variable haber estado más de dos años en desempleo disminuye las posibilidades de acceder al programa obtiene un efecto negativo y significativo (tabla 3).

Tabla 3

Estimación del propensity score (modelo propit)

\begin{tabular}{|ccc} 
& $\begin{array}{c}\text { dF/dx para un cambio } \\
\text { en la variable de 0 a 1 }\end{array}$ & Error estándar \\
\hline Edad & .0018 & .0064 \\
\hline Estudios & & \\
\hline Graduado Escolar/ EGB/ & .0126 & .1294 \\
ESO & .0478 & .1269 \\
\hline FPs & -.2368 & .1773 \\
\hline Universitarios & -.1116 & .2018 \\
\hline Cobran prestación & .2765 & .1941 \\
\hline Desempleo/ Paro & -.3479 & .3694 \\
\hline Mayores de 45 & .1788 & .1881 \\
\hline Mayores de 52 & .0199 & .2294 \\
\hline Ayuda familiar & .0038 & .2735 \\
\hline Otras & -.0002 & .006 \\
\hline Meses de experiencia laboral & .0229 & .1039 \\
\hline \multicolumn{1}{|l}{ Menos de 1 año } & $-.2897^{*}$ & .1260 \\
\hline Más de 2 años & & \\
\hline Number of obs 136 & & \\
\hline LR chi2(14) 35.91 & & \\
Log likelihood -67.654 & & \\
\hline \hline
\end{tabular}

Nota: * significatividad al $5 \%$

Tras el cálculo del propensity score, el emparejamiento o matching nos permite determinar el impacto del programa sobre las usuarias. Se han realizado los emparejamientos con cinco unidades de comparación y sólo con aquellos que estén en un radio de .05, para que los matches sean lo más semejantes posibles.

Mediante la aplicación de este método queremos medir los resultados (efecto medio del tratamiento sobre los tratados, ATT) en las variables resultado: acceso al empleo y tiempo trabajado.

A partir de las estimaciones no se puede afirmar que la participación en el Programa "Puesto a Puesto VII" se traduzca en un mayor acceso al empleo, ya que supera en $0.6 \%$ la de las beneficiarias a la del grupo control, no siendo significativamente distinta de cero. Tampoco se muestran efectos del programa sobre las variables duración del empleo (aunque es superior, no es estadísticamente significativa) (tabla 4). 
Tabla 4

Efecto medio del PaP VII sobre las usuarias con el método vecino más cercano

\begin{tabular}{lcc} 
& ATT & Error Estandar \\
\hline Acceso al empleo & .0068 & .1438 \\
Duración del empleo & 1.2443 & 1.2510 \\
\hline
\end{tabular}

** Significativo al 5\%, * significativo al $1 \%$

La comparativa de resultados con otras investigaciones que apliquen el matching, como método para evaluar políticas activas de empleo que incluyan formación y asesoramiento, es complicada ya que no tenemos constancia de su publicación. Los estudios tomados como referencia, evalúan diferentes políticas activas, efectos del tratamiento, y establecen horizontes temporales distintos, pero en términos globales nuestros resultados son coincidentes con las investigaciones de Arellano (2005) (aunque utiliza una metodología distinta) y el de Mato Díaz y Cueto Iglesias (2008) que evidencian efectos positivos a corto plazo en el acceso al empleo y en el tiempo trabajado.

\section{Conclusiones}

Consideramos necesario avanzar y superar las limitaciones existentes, promoviendo la evaluación de la eficacia de las políticas activas de empleo (cursos, prácticas orientadoras, programas mixtos que engloben formación y orientación, talleres de búsqueda activa de empleo), ya que todas esas acciones persiguen un objetivo común, favorecer la inserción laboral, objetivo que es necesario evaluar.

Los análisis realizados nos han permitido probar el principio de causalidad (Cueto Iglesias, 2005), mediante el cual conocemos hasta qué punto la participación en el programa "Puesto a Puesto VII" es la razón que explica el logro del acceso al empleo. A través del método propensity score matching hemos determinado el impacto del programa, a través del efecto medio del tratamiento, sobre los tratados en las variables resultado. Los resultados para el método utilizado (neigbour matching) evidencian un efecto positivo de la participación en el acceso al empleo $(0.6 \%)$ de las beneficiarias (aunque mínimo el efecto), pero este no es significativo. Así mismo, se constata que el tiempo de permanencia en el empleo de las usuarias es mayor que las del grupo control, pero no significativamente distinto de cero.

Somos consiente de las dificultades que supone la puesta en marcha de una evaluación, y sobre todo si se consideran las prácticas evaluadoras todavía ancladas en finalidades de rendición de cuentas y no para determinar las consecuencias sociales derivadas de las acciones ya que las políticas de inclusión social e inserción laboral sitúan a las personas en el centro de sus actuaciones.

Así mismo, es de destacar que los resultados, tanto positivos como negativos, de una política activa de empleo concreta no van a cambiar el modelo que rige en la Unión Europea, pero si estos resultados se dan en varios programas, sobre todo detectando sus debilidades, acabarán "haciendo mella en los responsables políticos y administrativos" (Ballart Hernández, 2005, p. 224). 
Por último consideramos que es necesario crear una mayor sinergia entre las políticas sociales y las políticas de empleo, consiguiendo así que las personas sean el centro de las actuaciones puestas en marcha, potenciando su integración en el mercado laboral y en la sociedad huyendo de los enfoques más "asistenciales" que rigen las actuales políticas de empleo.

\section{Bibliografía}

Abadie, A. e Imbens, G. W. (enero, 2006). Large sample properties of matching estimators for average treatment effects. Econométrica, 74(1), 235-267. Recuperado de http://www.hks.harvard.edu/fs/aabadie/smep.pdf

Affuso, A. (septiembre, 2010). Do public subsidies reduce credit rationing? A matching approach. Munich Personal Repec Archive, 24874.

Alonso-Borrego, C., Arellano, A., Dolado, J.J. y Jimeno, J.F. (2004). Eficacia del gasto en algunas políticas activas en el mercado laboral español. Madrid: Fundación Alternativas.

Alonso Ramos, E. (2005). Descripción e impacto de dos programas de empleo. Revista del Ministerio de Trabajo y Asuntos Sociales, 56, 165-175.

Alonso Ramos, E. (2008). Modelo organizativo y evaluación de programas de empleo. Revista del Ministerio de Trabajo y Asuntos Sociales, 71, 149-168.

Alujas Ruíz, J.A. y López Tamayo, J. (2006). Efecto de las políticas activas sobre el proceso de emparejamiento en el mercado de trabajo español. Revista asturiana de economía, 36, 77-95.

Anxo, D., Carcillo, S. y Erhel, C. (2001). Agregate impact analysis of active labour market policy in France and Sweden: a regional approach. En J. de Koning y H. Mosley (coords.), Labour market policy and unemployment (pp. 49-76). Londres: Edward Elgar.

Arellano, A. (febrero, 2005). Evaluating the effects of labour market reforms "at the margin" on unemployment and employment stability: the Spanish case. Working Paper, Economic Series, 5. Universidad Carlos III. Recuperado de https://sites.google. com/site/alfonsoarellano/pagina-de-inicio/investigacion

Arellano, A. (2006). Una revisión sobre los métodos de estudio y evaluación en las políticas activas de empleo. Working papers, Instituto Valenciano de Investigaciones Económicas, S.A. Recuperado de http://econpapers.repec.org/paper/iviwpasec/2006-06.htm.

Ayala, L. y Rodríguez, M. (2006). The Latin Model of Welfare: Do Insertion Contracts reduce long-term dependence? Labour economics, 13, 799-822.

Ayala, L. y Rodríguez, M. (2007). Barriers to employment and welfare duration. Journal of Policy Modeling, 29, 237-257.

Ayala, L. y Rodríguez, M. (febrero, 2011). Health-related effects of welfare-to-work policies: evidence from Spain. Trabajo presentado en el XVIII Encuentro de Economía de Economía Pública, Málaga.

Ballart Hernández, X. (2005). Política europea de empleo para mujeres inactivas: ¿cómo sabremos si estamos acertando? Ekonomiaz, 60(1), 210-231.

Berman, P. (1980). Thinking about programmed and adaptative implementation. En H. Ingrand y D. Mann, Why policies succed or-fail? (pp. 37-56) Londres: Sage. 
Blundell, R., Dearden, L. y Sianesi, B. (2004). Evaluating the impact of education on earning in the UK: models, methods and results from the NCDS. London: Centre for the Economics of Education. Recuperado de http://eprints.lse.ac.uk/19451/1/Evaluating_the_Impact_of_Education_on_Earnings_in_the_UK_Models,_Methods_and_ Results_from_the_NCDS.pdf

Boeri, T. y Burda, M.C. (1996). Active labour market policies, Jobs matching and the Czech miracle. European Economic Review, 40(3-5), 805-817.

Buendía Eisman, L., Expósito López, J. y Sánchez Martínez, M. (2012). Investigación evaluativa de programas de formación profesional para el empleo en el ámbito local. Revista de Investigación Educativa, 30(1), 161-179 .Recuperado de http://revistas. um.es/rie/article/view/115971

Cueto Iglesias, B. (2005). Políticas activas de mercado de trabajo. Tres análisis empíricos en un marco regional (Tesis inédita de doctorado). Universidad de Oviedo, Oviedo.

Cueto Iglesias, B. y Mato Díaz, F.J. (junio, 2004). El matching como técnica de evaluación de políticas: una aplicación a las políticas de fomento del empleo. Trabajo presentado al VII Encuentro de Economía Aplicada, Vigo. Recuperado de http://www.alde.es/ encuentros/anteriores/viieea/trabajos1.html

Cueto Iglesias, B. y Mato Díaz, F. J. (diciembre, 2005). Evaluación mediante matching de la formación ocupacional: un estudio de caso para España. Trabajo presentado al XXX Simposio de Análisis Económico, Murcia. Recuperado de http://www.webmeets. com/files/papers/SAE/2005/183/SAE\%202005\%20Cueto\%20\&\%20Mato.pdf

de Koning J. y Arent, M. (2001). The impact of active labour market policy on job hirings and unempoyment in the Netherlands. En J. de Koning y H. Mosley (cords.), Labour market policy and unemployment (pp.115-136). Londres: Edward Elgar.

de Miguel, M., San Fabián, J.L., Belver, J.L. y Argüelles, M.C. (2011). Evaluación de la satisfacción de los participantes en la formación profesional para el empleo. Relieve, 17(1). Recuperado de www.uv.es/RELIEVE/v17n1/RELIEVEv17n1_3.htm

de Miguel, M., San Fabián, J.L., Belver, J.L. y Argüelles, M.C. (mayo- agosto, 2012). Evaluación de la inserción laboral de los participantes en la formación profesional para el empleo de Asturias. Revista de Educación, 358, 599-616.

Dehejia, R.H. y Wahba, S. (2002). Propensity score matching methods for nonexperimental causal studies. The Review of economics and statistics, 84 (1), 151-161. Recuperado de http://users.nber.org/ rdehejia/papers/matching

Durán, C. (2004). Evaluación microeconométrica de las políticas públicas de empleo: aspectos metodológicos. Revista de Economía Aplicada, 170,107-133.

García Pérez, J.I. y Rebollo Sanz, Y.F. (2009a). The use of permanent contracts across Spanish regions: Do regional wage subsidies work? Investigaciones económicas, 33 (1), 97-130.

García Pérez, J.I. y Rebollo Sanz, Y.F. (2009b). Do wage subsidies affect the subsequent employment stability of permanent workers?: the case of Spain. Moneda $y$ Crédito, 228,65-103. Recuperado de http://www.eea-esem.com/files/papers/EEAESEM/2009/603/Rebollo-EEA.pdf

García Serrano, C. (2007). Las políticas del mercado de trabajo: desempleo y activación laboral. Política y Sociedad, 44(2), 135-151. Recuperado de http://revistas.ucm.es/index. php/POSO/article/view/POSO0707230135A 
García Serrano, C., Malo, M.A., Herranz, V. Davia, M.A. y Toharia, L. (2001). Do active labour market policies matter in Spain? En J. de Koning y H. Mosley (cords.), Labour market policy and unemployment (pp.137-160). Londres: Edward Elgar.

González-Blanch, F. (2010). Consecuencias de la crisis financiera y social sobre la gobernanza del Modelo Social Europeo y el papel de la evaluación. Revista Gestión y Análisis de Políticas Públicas, 4, 79-100. Recuperado de http://www.redalyc.org/ pdf/2815/281521696004.pdf

Herrador Buendía, F. (2008). Política social de Mercado de trabajo en España. El origen del proceso de europeización de la política de fomento del empleo. Valencia: Tirant lo Blanch.

Jornet, J.M., Perales, M.J., Suárez, J.M., Pérez Carbonell, M.A., Chiva, I., Ramos, G.,...,Sánchez Delgado, P. (2001). La evaluación de programas de formación: tipos de planes y algunas cuestiones metodológicas. Revista de Investigación Educativa, 19(2), 589-597.

Mato Díaz, F.J. (2002). La formación para el empleo. Una evaluación cuasi-experimental. Madrid: Cívitas.

Mato Díaz, F.J. y Cueto Iglesias, B. (2008). Efecto de las políticas de formación a desempleados. Revista de Economía aplicada, 46(16), 61-83. Recuperado de http://www. revecap.com/revista/numeros/46/mato_cueto.html

Meager, N. y Evans, C. (2002). The evaluation of active labor market measures for the long term unemployed. Geneva: Employment and training paper.

Meyer, B.D. (2000). Lessons from U.S. unemployment insurance experiment. Journal of economic literature, 33, 152-165.

Monclús, A. y Sabán, C. (2000). Formación y empleo: ¿Qué formación? En A. Monclús (coord.). Formación y empleo: enseñanza y competencias (pp. 307-322). Granada: Editorial Comarés.

Olmos Rueda, P. (2014). Competencias básicas y procesos perceptivos: factores claves en la formación y orientación de los jóvenes en riesgo de exclusión educativa y sociolaboral. Revista de Investigación educativa, 32 (2), 531-546. Recuperado de http:// revistas.um.es/rie/article/viewFile/181551/163321

Palma Martos, L., Borra Marcos, C., González Camacho, C. y Aguado Quintero, L.F. (febrero, 2009). Una evaluación de los programas de inserción laboral: el caso del programa Redes del Ayuntamiento de Sevilla. Trabajo presentado al XVI Encuentro de Economía Pública, Granada. Recuperado de http://dialnet.unirioja.es/servlet/ articulo?codigo $=2942182$

Raaum, O. y Torp, H. (2002). Labour market training in Norway- effect on earnings. Labour economics, 9(2), 207-247.

Rebollo Quintela, N. y Muñoz Cantero, J.M. (2013). An active labour market policy evaluation: the Puesto a Puesto Programme. Journal of Educational Sciences, 28(2), 92-101.

Richardson, K. y Berg, G.J. (2001). The effect of vocational employment training on the individual transition ate from unemployment to work. Swedish Economic Policy Review, 8, 175-213.

Rocha Sánchez, F. (diciembre, 2010). Reflexiones y propuestas para la reforma de las políticas activas de empleo en España. Estudios de la Fundación, 42, 1-49. Recuperado de http://www.1mayo.ccoo.es/nova/files/1018/Estudio42.pdf 
Smith, J.A. y Todd, P.E. (2005). Does matching overcome LaLonde's critique of nonexperimental estimators? Journal of Econometrics, 125, 305-353. Recuperado de https:// www3.nd.edu/ wevans1/class_papers/smith_todd_j_of_econometrics.pdf

Suárez Cano, P., Mayor Fernández, M. y Cueto Iglesias, B. (2011). How important is access to employment offices in Spain? An urban and non-urban perspective. Investigaciones regionales, 21, 119-140. Recuperado de http://www.redalyc.org/articulo. oa?id=28922059008

Zapico-Goñi, E. (2010). El uso de la evaluación como instrumento de gestión y gobernanza en la Unión Europea. Revista de Gestión y Análisis de Políticas Públicas. Nueva Época, 4, 101-133.

Fecha de recepción: 24/09/2014

Fecha de revisión: 26/09/2014

Fecha de aceptación: 07/08/2015 